\title{
Three-Input Majority Logic Gate and Multiple Input Logic Circuit Based on DNA Strand Displacement
}

\author{
Wei Li,* Yang Yang, Hao Yan, and Yan Liu* \\ Department of Chemistry and Biochemistry, and Center for Single Molecule Biophysics, Biodesign Institute at Arizona State \\ University, 1001 South McAllister Avenue, Tempe, Arizona 85287-5601, United States
}

\section{Supporting Information}

\begin{abstract}
In biomolecular programming, the properties of biomolecules such as proteins and nucleic acids are harnessed for computational purposes. The field has gained considerable attention due to the possibility of exploiting the massive parallelism that is inherent in natural systems to solve computational problems. DNA has already been used to build complex molecular circuits, where the basic building blocks are logic gates that produce single outputs from one or more logical inputs. We designed and experimentally realized a

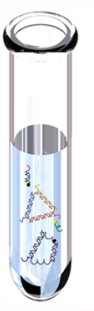

\begin{tabular}{cccc}
\hline A & B & C & Output \\
\hline 0 & 0 & 0 & 0 \\
1 & 0 & 0 & 0 \\
0 & 1 & 0 & 0 \\
0 & 0 & 1 & 0 \\
1 & 1 & 0 & 1 \\
1 & 0 & 1 & 1 \\
0 & 1 & 1 & 1 \\
1 & 1 & 1 & 1 \\
\hline
\end{tabular}

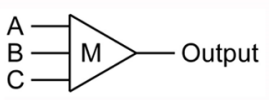

three-input majority gate based on DNA strand displacement. One of the key features of a three-input majority gate is that the three inputs have equal priority, and the output will be true if any of the two inputs are true. Our design consists of a central, circular DNA strand with three unique domains between which are identical joint sequences. Before inputs are introduced to the system, each domain and half of each joint is protected by one complementary ssDNA that displays a toehold for subsequent displacement by the corresponding input. With this design the relationship between any two domains is analogous to the relationship between inputs in a majority gate. Displacing two or more of the protection strands will expose at least one complete joint and return a true output; displacing none or only one of the protection strands will not expose a complete joint and will return a false output. Further, we designed and realized a complex five-input logic gate based on the majority gate described here. By controlling two of the five inputs the complex gate can realize every combination of OR and AND gates of the other three inputs.
\end{abstract}

KEYWORDS: Molecular programming, molecular computing, DNA strand displacement, majority logic gate

$T^{k}$ he ability to program interactions between biomolecules can help us to understand life processes and activities at the molecular level. DNA is an ideal candidate for molecular programming that facilitates both in vivo and in vitro applications ${ }^{1}$ because of its biological and physical properties. The behavior of DNA molecules with particular sequences can be reliably predicted according to the Watson-Crick basepairing principle. The recent developments in the field of structural DNA nanotechnology ${ }^{2}$ provide many different platforms onto which logically programmed DNA interactions can be combined and organized.

The first employment of DNA as molecular programming reagent resulted in a solution to the seven-city Hamilton path problem. ${ }^{3}$ Since then, several enzyme-catalyzed ${ }^{4-6}$ and enzymefree $^{7-10}$ DNA automata systems have been designed and realized. In the enzyme-free systems single-stranded DNA (ssDNA) molecules are used as input signals. Introducing the input signals to a system containing other double-stranded DNA (dsDNA) molecules displaying ssDNA toeholds results in a series of toehold directed strand displacement reactions ${ }^{11-15}$ and the release of an ssDNA molecule as a detectable output signal. Computing circuits based on DNA strand displacement that demonstrate complicated computations such as a binary square $\operatorname{root}^{16}$ and network computations ${ }^{17}$ were achieved with high efficiency and accuracy. In these computing circuits both AND and OR gates were utilized.

In this work we achieved the construction of a three-input majority logic gate by programming DNA interactions. A majority logic gate with multiple inputs returns true outputs, if and only if more than half of the inputs are true. A three-input majority gate is one of the most basic logic gates and has been demonstrated using magnetic quantum-dot cellular automata (MQCA). ${ }^{18}$ With multiple inputs this gate can accept and produce a high volume of information; thus, on the molecular level a three-input majority gate can serve as a basic and versatile building block for constructing more complex circuits. Here we experimentally realized a three-input majority gate with programmed DNA strand displacement reactions for the first time and demonstrated that it reliably produces all the correct outputs with different combinations of the inputs. We further constructed a five-input computing circuit implemented solely by linking two three-input majority gates together. This circuit can be tuned to accomplish four different computing patterns among the various combinations of the inputs.

Received: May 2, 2013

Revised: $\quad$ May 23, 2013

Published: May 27, 2013 

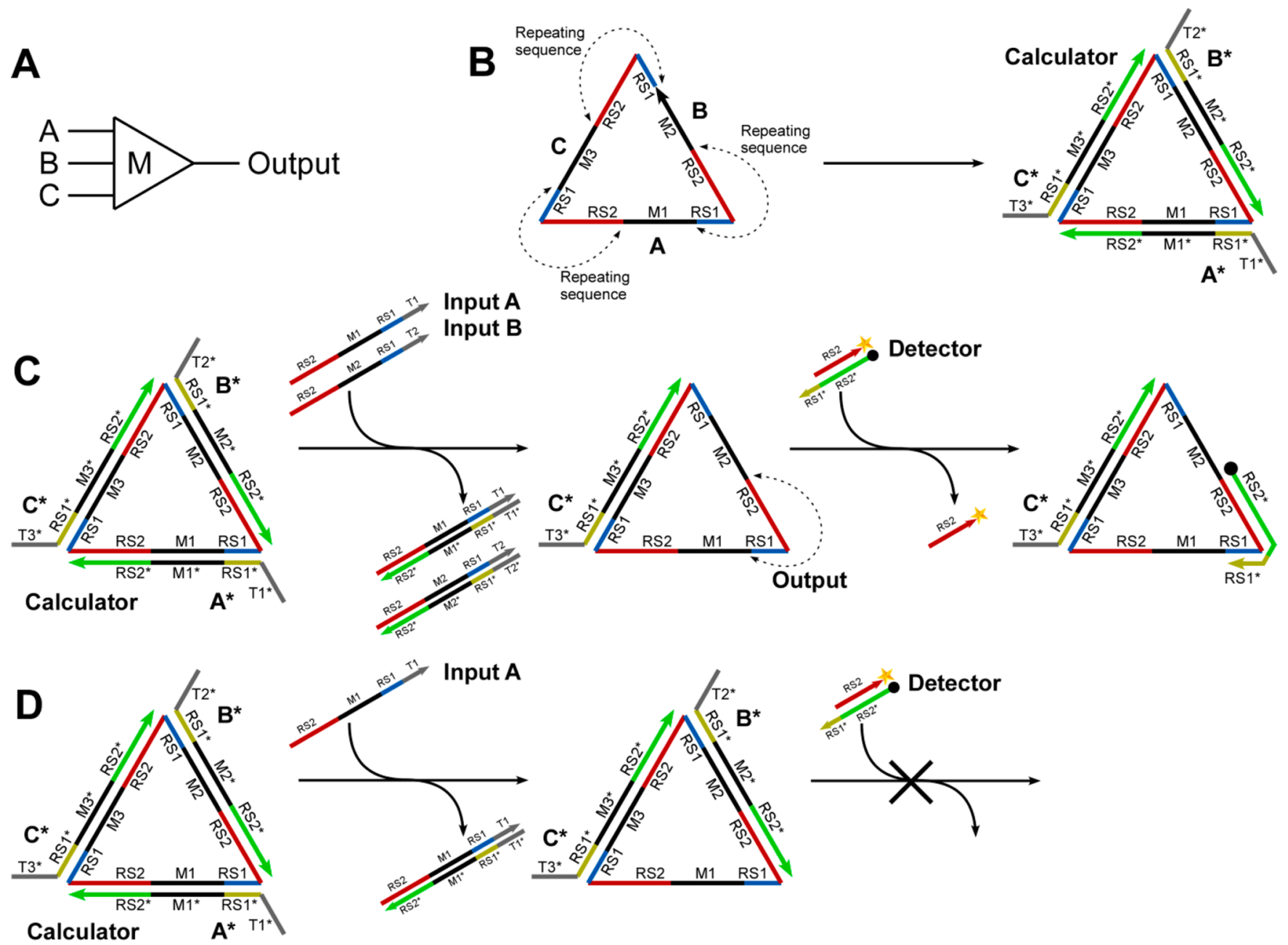

Figure 1. Architectural design of a three-input majority gate based on DNA strand displacement reactions. (A) Symbolic representation of the majority logic gate. (B) Design of the Calculator structure. The circular ssDNA (left) is composed of three segments, A (RS2-M1-RS1), B (RS2-M2RS1), and C (RS2-M3-RS1). Each segment has 42 nts. RS1 and RS2 are 8 and 18 nts long, respectively. The RS1-RS2 joint sequence is repeated in the circular structure three times. M1, M2, and M3 are each 16 nts long and have distinct sequences. ssDNA $\mathrm{A}^{*}, \mathrm{~B}^{*}$, and $\mathrm{C}^{*}$ are hybridized with $\mathrm{A}$, $\mathrm{B}$, and $\mathrm{C}$, respectively, forming the Calculator structure (right). $\mathrm{A}^{*}, \mathrm{~B}^{*}$, and $\mathrm{C}^{*}$ each have two domains: one domain, $\mathrm{RS} 1^{*}$-M1 $(2,3) *-\mathrm{RS} 2^{*}$, is fully complementary to A, B, or C. The other domain, $\mathrm{T} 1(2,3)^{*}$, is a unique sequence toehold for initiating the computation process. (C) Two or three inputs lead to a true output. Here a representative two-input model is shown. Input A and input B are fully complementary to $A^{*}$ and $B^{*}$, respectively. The toeholds, $\mathrm{T} 1$ and $\mathrm{T} 2$, first hybridize with $\mathrm{T} 1^{*}$ and $\mathrm{T} 2 *$. Next, the input strands fully displace $\mathrm{A}^{*}$ and $\mathrm{B}^{*}$ from the circular structure. Finally $\mathrm{A}^{*}$ and $\mathrm{B}^{*}$ hybridize with Input A and Input B and are displaced from the Calculator. The RS1-RS2 joint (output) on the circular strand is then fully exposed, yielding a true output. A "Detector" is premixed with the Calculator. The Detector is a duplex of RS2 and RS2*-RS1*. RS2 is modified with 6-carboxyfluorescein (FAM) at the $3^{\prime}$ end. RS2*-RS1* is modified with Iowa Black dark quencher (IABk) at the $5^{\prime}$ end. RS1 in the output and RS1* in the Detector serve as toeholds, and RS2-FAM is displaced from the dark quencher; thus the true output is revealed by a fluorescence increase. (D) One or no input leads to a false output. A representative 1 input (e.g., Input A) case is shown. Only $A^{*}$ is released by Input A; thus no continuous RS1-RS2 is exposed, and the output is 0 . The Detector duplex is highly stable, and the fluorescence remains quenched through the computation process.

Architecture Design. Single Three-Input Majority Gate. For a three-input majority gate (see Figure 1A), if any two or all of the three inputs are true, the output is true. The truth table (Table 1) specifies that the three inputs have the same priority among one another. Thus, for a three-input majority gate the outputs between any combinations of two or three inputs should not be distinguishable. To construct a three-input majority gate from DNA molecules we implemented a circular DNA strand consisting of three distinct segments, A, B, and C (Figure 1B); in each segment the middle portion is unique (M1, M2, and M3, 16 nts each), and the three joints are identical (RS2 $18 \mathrm{nts}$ and RS1, 8 nts). Before performing the computation segments $\mathrm{A}, \mathrm{B}$, and $\mathrm{C}$ each hybridize to a complementary ssDNA molecule $\left(\mathrm{A}^{*}, \mathrm{~B}^{*}\right.$ and $\mathrm{C}^{*}$, respectively)
Table 1. Truth Table of a Three-Input Majority Logic Gate

\begin{tabular}{cccc} 
input A & input B & input C & output \\
0 & 0 & 0 & 0 \\
1 & 0 & 0 & 0 \\
0 & 1 & 0 & 0 \\
0 & 0 & 1 & 0 \\
1 & 1 & 0 & 1 \\
1 & 0 & 1 & 1 \\
0 & 1 & 1 & 1 \\
1 & 1 & 1 & 1 \\
\hline
\end{tabular}

forming a circular (quasi-triangular) duplex. Strands $A^{*}, B^{*}$, and $C^{*}$ each have two domains: one domain is fully 
complementary to $\mathrm{A}, \mathrm{B}$, and $\mathrm{C}$, respectively, and the other domain displays a toehold ( $\mathrm{T} 1 *, \mathrm{~T} 2 *$, and $\mathrm{T} 3 *, 10 \mathrm{nts}$ each) for initiating the strand displacement reaction. This circular duplex structure is referred to as a "Calculator" herein.

Three unique input strands (inputs $\mathrm{A}, \mathrm{B}$, and $\mathrm{C}$ ) are designed to be fully complementary to $\mathrm{A}^{*}, \mathrm{~B}^{*}$, and $\mathrm{C}^{*}$ (both domains). When the inputs are introduced to the computing system toehold-mediated strand displacement reactions are initiated. For those cases in which there are two or three inputs (i.e., majority input; Figure 1C), ssDNA from two or three sides of the Calculator are released. The release events expose a single joint (for two inputs) or all three joints (for three inputs), in the Calculator structure, and segments $\mathrm{A}, \mathrm{B}$, and $\mathrm{C}$ is/are concurrently exposed as ssDNA. The exposure of at least one joint domain (all with the same sequence) is defined as a positive output. A "Detector" is utilized to recognize and report the output. The detector is composed of two strands that form a duplex displaying a toehold and is labeled with a fluorescence dye and a corresponding dark quencher on the two component strands. The strand that is modified with the dark quencher carries the toehold that is fully complementary to the output. When it hybridizes with the output, the fluorescence-dyemodified strand is released to the solution, and an increase in the fluorescence intensity of the dye is detected as proof of a true output.

When only one or no input (minority input) is introduced (Figure 1D), none of the joint domains of the three segments is fully exposed. Even though two joint domains may be partially exposed, because they are operating separately at opposite ends of a segment they cannot disassemble the detector duplex, and the output remains 0 .

Logic Gate Cascade. As indicated by the truth table (Table 1 ), an important property of a three-input majority gate is that, if any of the three inputs is preset as 1 , the logic gate becomes an OR gate for the remaining two inputs (Figure 2A), and if

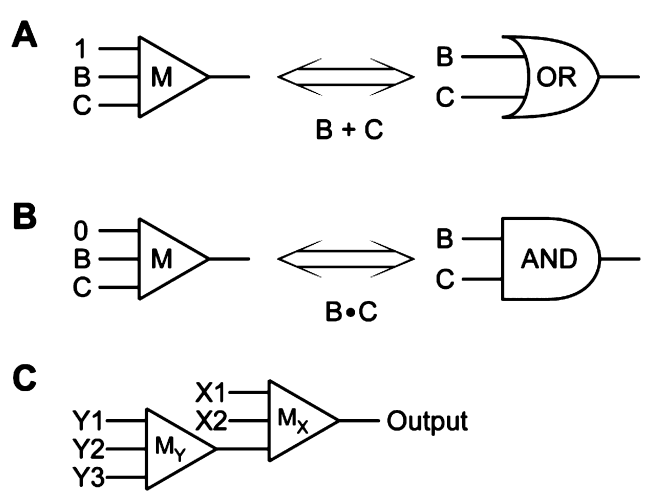

Figure 2. Properties of a three-input majority gate and the design of a multifunctional circuit. (A) If any one of the three inputs of a majority gate is preset as 1 , the gate becomes an OR gate of the remaining two inputs. In the figure, input $A$ is preset as 1 . The relationship between Inputs $\mathrm{B}$ and $\mathrm{C}$ becomes an OR function $(\mathrm{B}+\mathrm{C})$. (B) If any one of the three inputs of a majority gate is preset as 0 , the gate becomes an AND gate of the remaining two inputs. In the figure, input $A$ as 0 is shown. The relationship between inputs $B$ and $C$ becomes an AND function (B.C). (C) The multifunctional circuit contains two majority gates and has a depth of 2 . The output of the first generation, Majority Gate $Y\left(M_{Y}\right)$, is employed as one input of the second generation, Majority Gate $\mathrm{X}\left(\mathrm{M}_{\mathrm{X}}\right)$. There are five inputs of the circuit: $\mathrm{Y} 1, \mathrm{Y} 2$, and $\mathrm{Y} 3$ to $\mathrm{M}_{\mathrm{Y}} ; \mathrm{X} 1$ and $\mathrm{X} 2$ to $\mathrm{M}_{\mathrm{X}}$. The output of the second generation is the output of the entire circuit. any of the three inputs is preset as 0 , the logic gate becomes an AND gate for the remaining two inputs (Figure 2B). This ability to switch between OR and AND gates makes the threeinput majority gate a versatile building block for constructing more complex computing circuits.

To demonstrate switching of a three-input majority gate we assembled a computing circuit composed of two majority gates arranged sequentially (Figure $2 \mathrm{C}$ ). Majority Gate $\mathrm{Y}\left(\mathrm{M}_{\mathrm{Y}}\right)$ is the first generation gate. The output of $\mathrm{M}_{\mathrm{Y}}$ is utilized as one of the inputs of Majority Gate $X\left(M_{X}\right)$, which is the second generation gate. The output from $M_{X}$ is read as the final output of the circuit. The circuit has five inputs in total: Y1, Y2, and Y3 in $M_{Y} ; X 1$ and $X 2$ in $M_{X}$. By assigning values of 0 or 1 to any one of the inputs in each majority gate, this circuit can be switched between four different computing patterns for the remaining three inputs (Table 2). These four logical computing patterns represent all of the combinations of OR and AND functions between the three inputs.

Table 2. Computing Patterns of the Multifunctional Circuit under Different Preset Values of X1 and Y1

\begin{tabular}{ccc}
$\mathrm{X} 1$ & $\mathrm{Y} 1$ & computation pattern \\
0 & 0 & $\mathrm{Y} 2 \cdot \mathrm{Y} 3 \cdot \mathrm{X} 2$ \\
1 & 0 & $\mathrm{Y} 2 \cdot \mathrm{Y} 3+\mathrm{X} 2$ \\
0 & 1 & $(\mathrm{Y} 2+\mathrm{Y} 3) \cdot \mathrm{X} 2$ \\
1 & 1 & $\mathrm{Y} 2+\mathrm{Y} 3+\mathrm{X} 2$ \\
\hline
\end{tabular}

Based on the success of the single majority gate design described above (shown in Figure 1C,D), we engineered a twogeneration circuit as shown in Figure 3. Similarly, the Calculator structures in both generations feature a circular (quasi-triangular) design. The sequences of the joint domains between any two arms of Calculator Y (first generation) are all the same such that fully exposing any of the joints results in a true output of $\mathrm{M}_{\mathrm{Y}}$. Each joint domain is fully complementary to arm X3 in Calculator X (the second generation). Therefore, the output of $\mathrm{M}_{\mathrm{Y}}$ acts as an intermediate of the circuit and can be used as an input for the next generation calculator. For example, if the output of $M_{Y}$ is true, $M_{X}$ receives a true input from $M_{Y}$; and if the output of $M_{Y}$ is false, $M_{X}$ receives a false input from $M_{Y}$. Depending on the output of $M_{Y}$ and the additional two inputs of $\mathrm{M}_{\mathrm{X}}$ (X1 and $\mathrm{X} 2$ ) Calculator $\mathrm{X}$ produces the final output of the circuit. For example, if any two or three of the inputs for $\mathrm{M}_{\mathrm{X}}$ are present, two or three of the arm strands $\left(\mathrm{X}^{*}, \mathrm{X} 2 *\right.$, and $\left.\mathrm{X} 3 *\right)$ are displaced from Calculator $X$, exposing at least one joint domain of the circular strand as ssDNA which yields a true final output. Conversely, if only one or none of the inputs for $M_{X}$ is present, the final output is false. The output reacts with the "Detector", binding with the dark quencher labeled strand and releasing the fluorescence of the dye modified strand. The output is visualized by an increase in the fluorescence intensity of the dye, following the same mechanism as for the single majority gate.

Results and Discussion. Assembly of the Calculators. The central circular ssDNA molecules (126 nts long for the single three-input majority gate, $159 \mathrm{nts}$ long for $\mathrm{M}_{\mathrm{Y}}$, and $96 \mathrm{nts}$ for $\mathrm{M}_{\mathrm{X}}$ ) in the calculators are prepared by ligating one or two linear ssDNAs end to end (Figure S1). T4 DNA ligase is used to catalyze the circularization reactions. The termini of the ssDNA fragments are specifically paired and joined by hybridizing to 20-nt ssDNA templates, and the resulting nicks are then sealed with T4 DNA ligase. The circular ssDNA is 


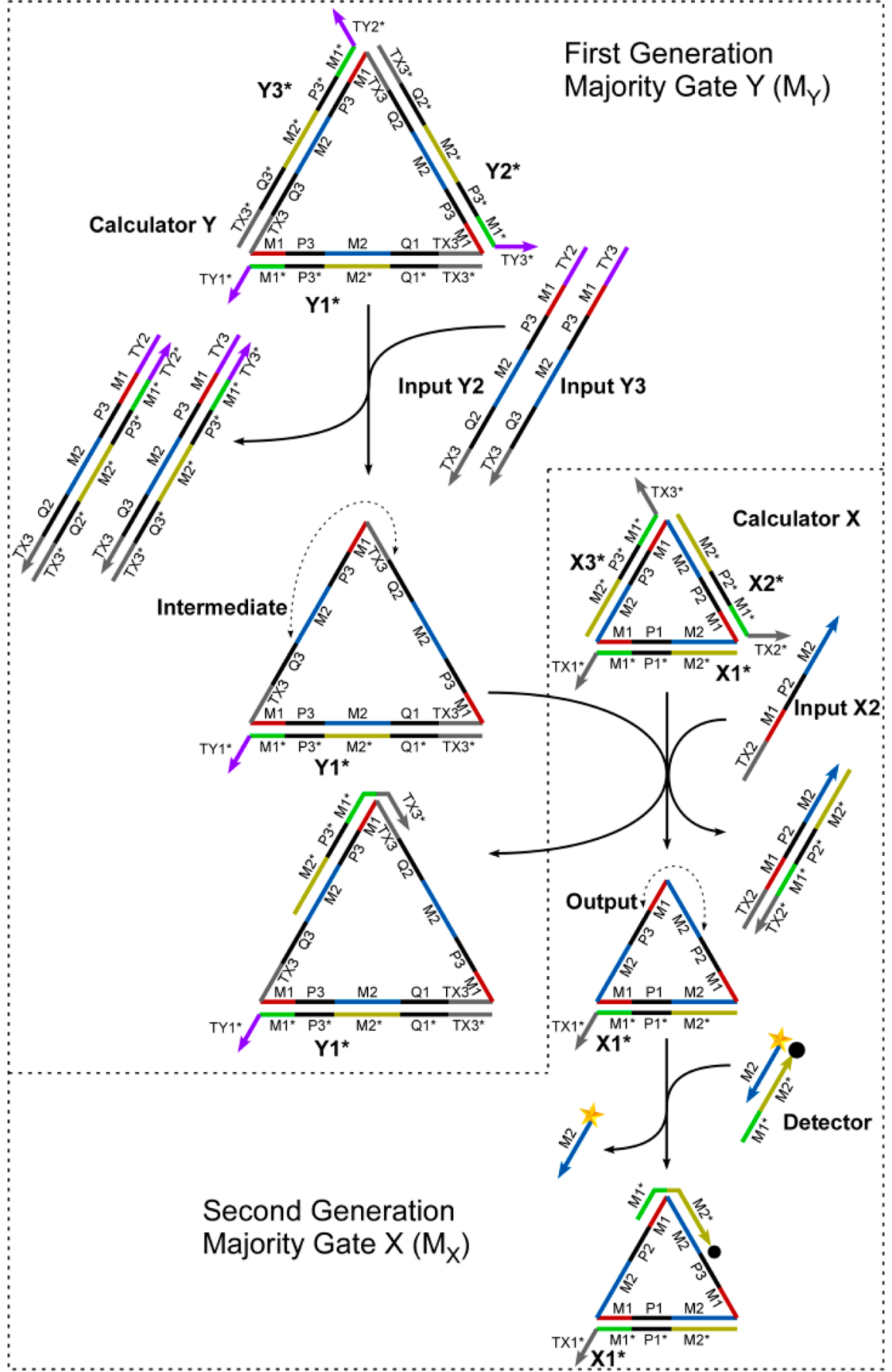

Figure 3. Designed reaction flow of the multifunctional circuit. Majority Gate $Y\left(M_{Y}\right)$, the first generation in the circuit, is shown in the upper-left. Majority Gate $X\left(M_{X}\right)$, the second generation in the circuit, is shown in the lower-right. For $\mathrm{M}_{\mathrm{Y}}$, there are three segments in circular Calculator $\mathrm{Y}$, each with three domains. One domain of the circular strand is M1-P3-M2. A second domain is Q1(2,3); Q1, Q2, and Q3 are each a unique sequence. The third domain is TX3. The three joint segments of the circular strand are all TX3-M1-P3-M2. Once Calculator Y has two or three arms displaced, it will return a true $\mathrm{M}_{\mathrm{Y}}$ output, exposing the Intermediate (TX3-M1-P3-M2) as ssDNA. This Intermediate is fully complementary to arm strand X3* in Calculator X and can therefore serve as an input of $\mathrm{M}_{\mathrm{X}}$. TX3 in the Intermediate functions as a toehold, and M1-P3-M2 displaces the remainder of $\mathrm{X} 3$ * from Calculator $\mathrm{X}$. For $\mathrm{M}_{\mathrm{X}}$, there are also three segments in Calculator $\mathrm{X}$. The design of Calculator $\mathrm{X}$ is similar to the design of the single gate shown in Figure 1, except for the length of each domain. The intermediate and the other two inputs of $\mathrm{M}_{\mathrm{X}}$, input X1 and input X2, determine the output of the overall circuit. The ssDNA output signal is the repeating joint sequence of the circular strand in Calculator X, M2-M1. Similar to the single gate design, the output of the circuit can be detected via changes in the fluorescence of a dye molecule. A representative computing pattern example is shown in the figure. Input $\mathrm{Y} 1$ is preset as 0 , which means that no ssDNA input $\mathrm{Y} 1$ is introduced to the reaction. Input $\mathrm{X} 1$ is also preset as 0 . As a result of the preset values of inputs $\mathrm{Y} 1$ and $\mathrm{X} 1$, the computing pattern in the figure is $\mathrm{Y} 2 \cdot \mathrm{Y} 3 \cdot \mathrm{X} 2$. Inputs $\mathrm{Y} 2$, $\mathrm{Y} 3$, and $\mathrm{X} 2$ are all present in the reaction system so the logical computing result is $1 \cdot 1 \cdot 1=1$. The lengths (in base pairs) of the domains in the figure: $\operatorname{TX1}(2,3)=\operatorname{TX1}(2,3) *=10$. $\mathrm{P} 1(2,3)=\mathrm{P} 1(2,3) *=9 . \mathrm{M} 1=\mathrm{M} 1 *=8 . \mathrm{M} 2=\mathrm{M} 2 *=15 . \operatorname{TY} 1(2,3)=\operatorname{TY} 1(2,3) *=10 . \mathrm{Q} 1(2,3)=\mathrm{Q} 1(2,3)^{*}=11$.

purified and recovered by denaturing polyacrylamide gel electrophoresis (PAGE). The overall recovery yield of the purified circular ssDNA is $30-50 \%$ (Figure S2A); note that the circularized strands are resistant to degradation by exonuclease 


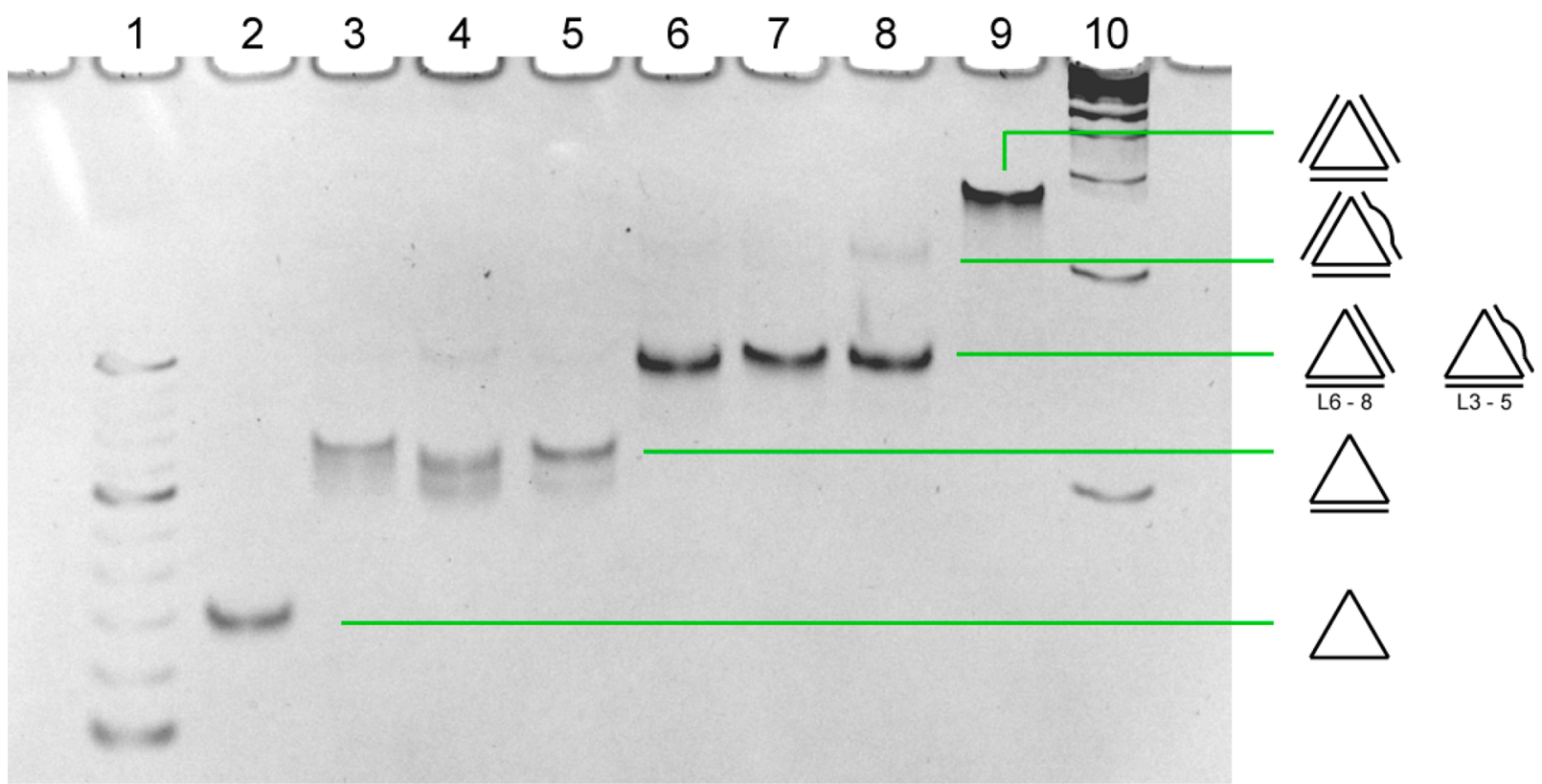

Figure 4. Native polyacrylamide gel electrophoresis confirming the formation of the single gate Calculator of the single gate. Lane 1: 10 bp DNA ladder. The three intense bands are $50 \mathrm{bps}, 100 \mathrm{bps}$, and $150 \mathrm{bps}$ from bottom to top, respectively. Lane 2: center circular strand. Lanes 3-5: center strand with one arm strand: $A^{*}, B^{*}$, or $C^{*}$, respectively. Lanes 6-8: center strand with two arm strands: $A^{*}+B^{*}, A^{*}+C^{*}$, or $B^{*}+C^{*}$, respectively. Lane 9: center strand with three arm strands: $A^{*}+B^{*}+C^{*}$, forming the complete Calculator structure. Lane 10: 100 bp DNA ladder. For each segment of the center circular strand, the two termini are portions of the repeating sequence. As a result, if a segment of the center strand does not have a fully complementary arm strand present in the system, its two ends may hybridize with the excess arm strands intended to interact with other segments such that with the middle portion of the segment is not bound. This process may result in species with retarded mobility as shown in Lanes $3-8$.

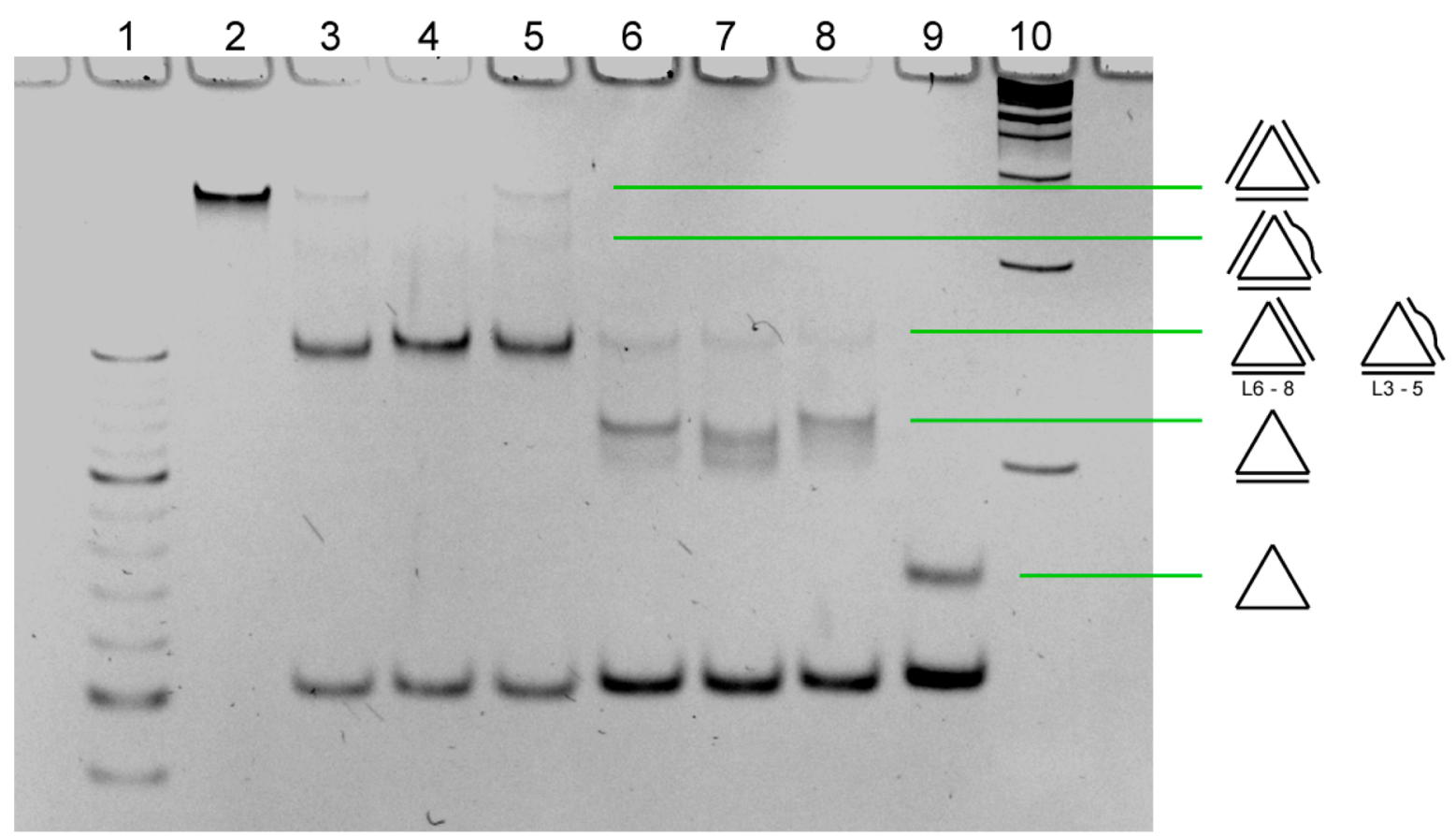

Figure 5. Native PAGE demonstrating the single gate design Calculator. Lane 1: $10 \mathrm{bp}$ DNA ladder. The three intense bands are $50 \mathrm{bps}, 100 \mathrm{bps}$ and 150 bps from bottom to top, respectively. Lane 2: the fully assembled Calculator. Lanes 3-5: the Calculator with a single input: Inputs A, B, or C respectively. Lanes 6-8: the Calculator with two inputs: Inputs A + B, Inputs A + C, or Inputs B + C, respectively. Lane 9: the calculator with all three inputs. Lane 10: $100 \mathrm{bp}$ DNA ladder. For each segment of the center circular strand, the two termini are portions of the repeating sequence. As a result, if a segment of the center strand does not have a fully complementary arm strand present in the system, its two ends may hybridize with the excess arm strands intended to interact with other segments such that with the middle portion of the segment is not bound. This process may result in species with retarded mobility as shown in Lanes $3-8$.

I (Figure S2B). The purified central circular ssDNA is hybridized with the three arm strands, forming the Calculator
(Figure 4). The molar ratio between the circular ssDNA and each arm strand is $1: 1.1$. 

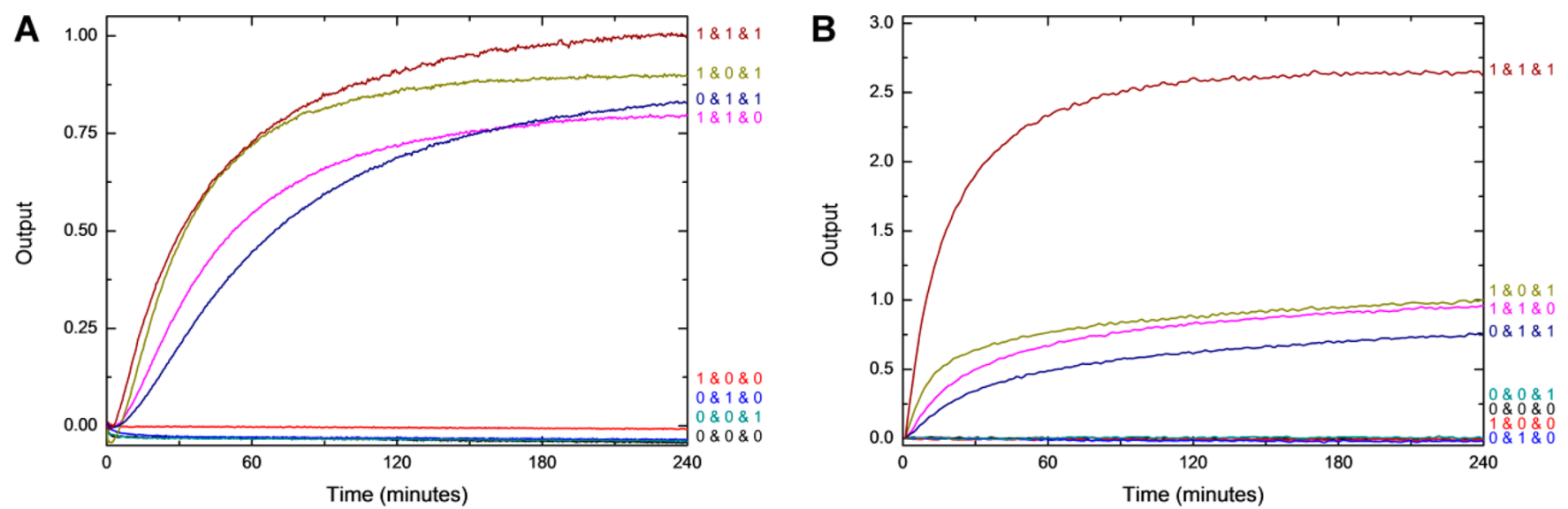

Figure 6. Kinetic characterization of the single three-input majority gate. (A) The ratio between the Detector and the Calculator is 1:1. (B) The ratio between the Detector and the Calculator is 4:1. Each curve in these two graphs represents a reaction corresponding to the inputs specified next to each curve. The fluorescence measurement begins at the moment that the Calculator, the Detector, and the inputs of each reaction are mixed. The fluorescence intensity is collected every $0.5 \mathrm{~min}$. The fluorescence increase is calculated by subtracting the initial intensity from the final intensity, normalized and plotted. For a 1:1 or 4:1 Detector to Calculator ratio, single or no input cases all return an output of 0 . The two or three input cases all return an output above 0.75 . The three inputs case for a 4:1 Detector to Calculator ratio returns an output of 2.7 , very close to the theoretically predicted value of 3 .

Gel Characterization of Calculator Formation and Operation with Inputs. The Calculators are prepared with excess arm strands that do not need to be removed before use. After a Calculator is prepared the specific input strands are mixed with the Calculator at a molar ratio of 1.2:1. The input strands displace the arm strands from the central circular strand of the Calculator. The structural changes of the Calculator corresponding to single gate reactions were characterized by native PAGE (Figure 5).

The gel image shown in Figure 5 clearly demonstrates the difference between true and false outputs of the logic gate for different input combinations. Lane 2 corresponds to no inputs, and the intact Calculator migrates as a single band. Lanes 3, 4, and 5 correspond to systems with a single input. Multiple bands are present in the gel image, but the emergence of species with fully exposed circular strand joints was not observed. Lanes 69 correspond to systems with two or three inputs where at least one output ssDNA is evident.

Detecting the Operation of a Single Majority Gate. A fluorescent dye molecule was used to detect the products of the three-input majority gate and to follow the kinetics of the logic computing reactions (Figure 6). The Calculator, a specific combination of inputs, and the FAM-Iowa Black modified Detector are mixed, and the fluorescence intensity of FAM (Ex $490 \mathrm{~nm}, \mathrm{Em} 520 \mathrm{~nm}$ ) is measured every $30 \mathrm{~s}$ at a constant temperature of $\sim 20^{\circ} \mathrm{C}$.

At the beginning of the reaction the fluorescence intensities of all input combinations are low because the FAM modified strand in the detector remains hybridized to the dark quencher modified strand. For reactions with one or no input, no output ssDNA is produced as the reaction proceeds. Thus, the FAM strands are never released from interaction with the dark quencher. The fluorescence intensities of these reactions remain at a low level throughout the experiment, indicating a false output of the majority logic gate.

For reactions with two or three inputs, one or three ssDNA output domains of the Calculators are exposed. The outputs are subsequently recognized by the Detector through toehold hybridization events. Next, the output displaces the FAM modified strand (toehold mediated displacement) from the dark quencher modified strand. As a result the fluorescence intensity increases, indicating the true output. The reaction rates are high at the initial stages of the reaction and slow down considerably as more and more Calculator species and ssDNA inputs are consumed. After the reaction reaches equilibrium, the fluorescence intensity of that system remains constant. The computation of each input combination finishes in $0.5-1.5 \mathrm{~h}$.

From the design shown in Figure 1 it is apparent that, if all three inputs are introduced to the Calculator, three ssDNA output domains would be exposed. Therefore, the molar ratio between the output and the Calculator is 3:1. However, for the three cases with combinations of two inputs the molar ratio between the output species and the Calculator is $1: 1$ because there is only one output domain exposed per Calculator. Thus, when there is 3 or more fold excess of the Detector present, the final fluorescence intensity of the three-input model is expected to be three times higher than the two-input cases (Figure 6B). If the amount of the Detector in the system is decreased to the same level as that of the Calculator, the final fluorescence intensities of the true output cases will be limited by the availability of the Detector. Figure 6A illustrates such a scenario in which four true outputs yield similar fluorescence intensity levels. The reaction kinetics is the fastest for the system with all three inputs, and for the two-input systems the rates are similar when input $B$ is absent, but become much slower when either input $\mathrm{A}$ or $\mathrm{C}$ is missing. The 1 - to 2 -fold difference in the reaction kinetics is not well understood. We speculate that it may originate from sequence-specific interactions between the DNA strands, especially in the toehold regions.

The raw data collected from the fluorescence experiments is the absolute intensity of the detector bound dye at each time point in the reaction. The fluorescence increase for each reaction is calculated by subtracting the initial intensity from the final intensity. For cases with a 1:1 Detector-to-Calculator ratio, the fluorescence increase is normalized to 1 (Figure 6A). The curves corresponding to reactions with two or three inputs plateau above 0.75 , while the curves with one or no input reach an equilibrium very close to 0 .

For cases with a 4:1 Detector-to-Calculator ratio, the fluorescence increase is normalized to the largest intensity 
A

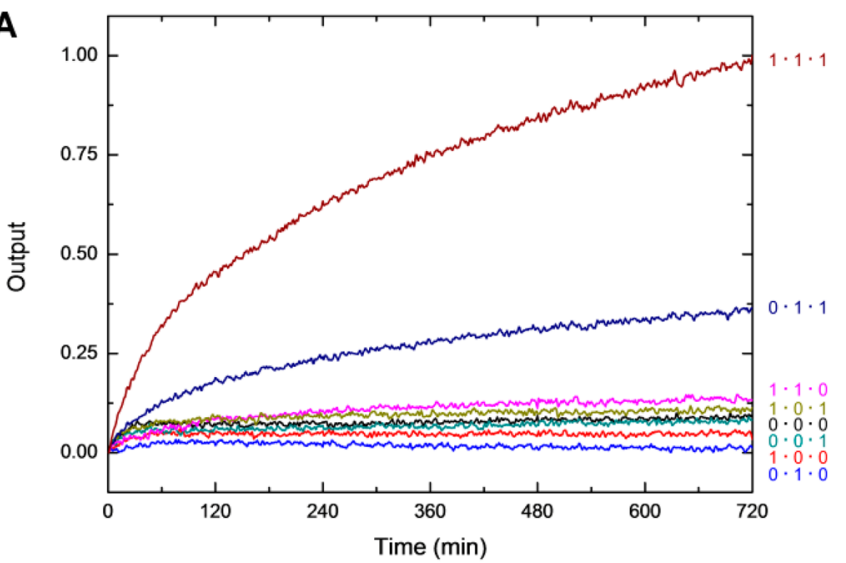

C

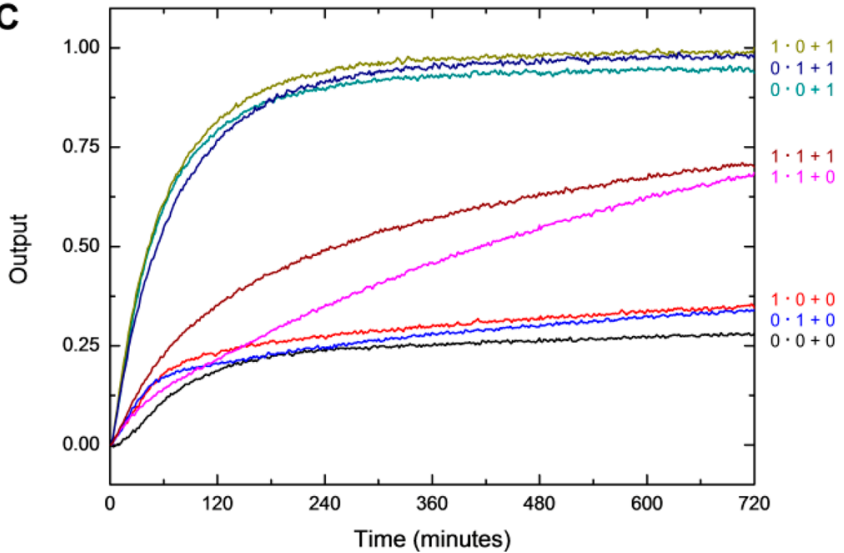

B

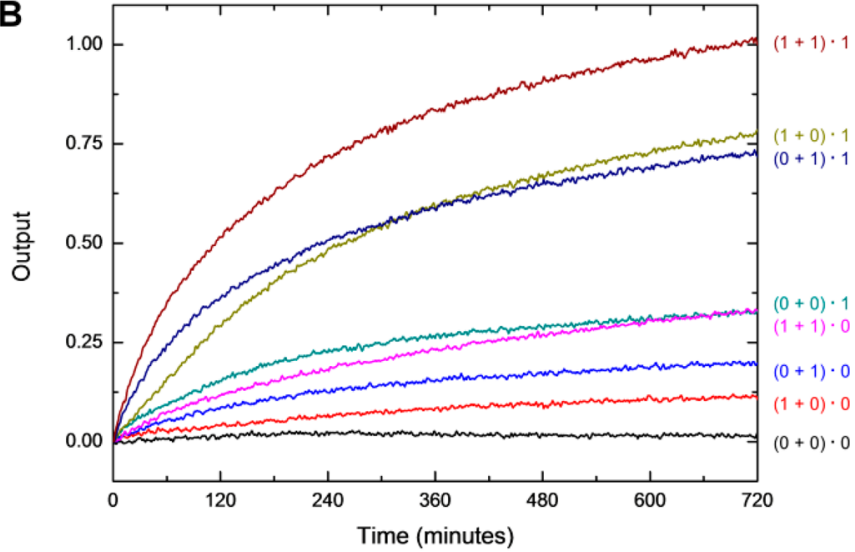

D

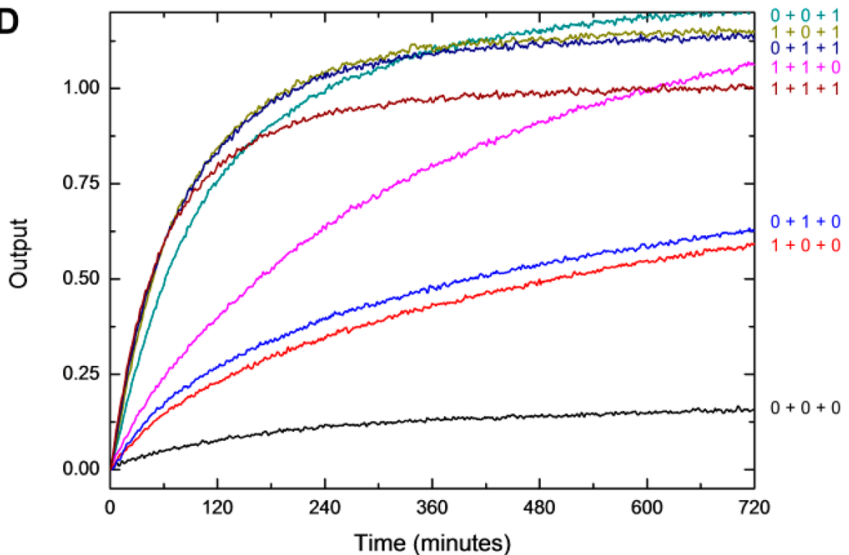

Figure 7. Kinetic characterization of the multifunctional circuit composed of two three-input majority gates. (A) Input $\mathrm{Y} 1$ is preset as 0 ; input $\mathrm{X} 1$ is preset as 0 . The computation pattern is $\mathrm{Y} 2 \cdot \mathrm{Y} 3 \cdot \mathrm{X} 2$. Only when $\mathrm{Y} 2$, Y3, and $\mathrm{X} 2$ are all true does the circuit return true. (B) Input $\mathrm{Y} 1$ is preset as 1 ; input $\mathrm{X} 1$ is preset as 0 . The computation pattern is $(\mathrm{Y} 2+\mathrm{Y} 3) \cdot \mathrm{X} 2$. Three input combinations return true outputs. (C) Input $\mathrm{Y} 1$ is preset as 0 ; input $\mathrm{X} 1$ is preset as 1 . The computation pattern is $\mathrm{Y} 2 \cdot \mathrm{Y} 3+\mathrm{X} 2$. Five input combinations return true outputs. (D) Input $\mathrm{Y} 1$ is preset as 0 ; input $\mathrm{X} 1$ is preset as 1 . The computation pattern is $\mathrm{Y} 2+\mathrm{Y} 3+\mathrm{X} 2$. Seven input combinations return true outputs. Only when $\mathrm{Y} 2$, $\mathrm{Y} 3$, and $\mathrm{X} 2$ are all false does the circuit return false. Each curve in these four graphs represents a reaction where the input combination is labeled at the end of the curve. The fluorescence measurement begins at the moment that the Calculator, the Detector, and the inputs of each reaction are mixed. The fluorescence intensity is measured every minute. The fluorescence increase is calculated by subtracting the initial intensity from the final intensity, normalized and plotted.

increase of the two-input reactions (Figure 6B). Notably, the curve corresponding to the three-input reaction plateaus at more than 2.5 , while the curves corresponding to the two-input reactions all plateau around 1 . These results, in accordance with the three-input majority gate truth table, validate that our DNA based logic gate functions as designed.

Assembling a Multifunctional Circuit. Based on the success of the single three-input majority gate, we went on to construct a two-generation majority gate circuit. The circuit is composed of two majority gates operated in series (Figure 3). These two majority gates were individually verified, and the kinetics were examined (Figure S4). As shown in Table 2, by presetting one input in each gate of the circuit (Y1 and X1, for example), the circuit can realize four different computational patterns depending on the identities of the preset inputs. For each computation pattern there are eight unique operations, depending on the combinations of the other three inputs. Figure 7 presents the kinetics of these computing systems with different input combinations, with each panel of graphs representing one computing pattern. In each panel the fluorescence output versus time plots represent the reaction kinetics of a combination of inputs (specified next to each curve). The specific combinations of inputs are represented by three numbers that correspond to $\mathrm{Y} 2, \mathrm{Y} 3$, and $\mathrm{X} 2$, respectively. The output of $\mathrm{Y} 2$ and $\mathrm{Y} 3$ serves as the intermediate that passes information from the first generation $\left(M_{Y}\right)$ to the second generation $\left(\mathrm{M}_{\mathrm{X}}\right)$. For example, the operation $1+1+0$ implies the following information: (1) the relationship between $\mathrm{Y} 2$ and $\mathrm{Y} 3$ is "OR", which only occurs when input $\mathrm{Y} 1$ is preset as 1 ; (2) the relationship between (Y2 + Y3) and $\mathrm{X} 2$ is "OR", which only occurs when input $\mathrm{X} 1$ is preset as 1 ; (3) the intermediate between the two generations is the result of $\mathrm{Y} 2+\mathrm{Y} 3=1+1=$ 1 . Therefore, the expected final output is 1 . In another example, the operation $(1+0) \cdot 1$ implies the following information: (1) the relationship between Y2 and Y3 is "OR", which only occurs when input $\mathrm{Y} 1$ is preset as 1 ; (2) the relationship between (Y2 $+\mathrm{Y} 3$ ) and $\mathrm{X} 2$ is "AND", which only occurs when input $\mathrm{X} 1$ is preset as 0 ; (3) the intermediate between the two generations is the result of $\mathrm{Y} 2+\mathrm{Y} 3=1+0=1$. Here, the final output is 1 .

Figure 7A depicts the results of presetting both $\mathrm{Y} 1$ and $\mathrm{X} 1$ as 0 . Thus, the circuit functions as $\mathrm{Y} 2 \cdot \mathrm{Y} 3 \cdot \mathrm{X} 2$. For all the input combinations of $\mathrm{Y} 2, \mathrm{Y} 3$, and $\mathrm{X} 2$, only the system in which all three inputs are true returns a true output. The other seven input combinations should all return false. We experimentally confirmed this for all situations, except for $0 \cdot 1 \cdot 1$, where we 
observed minimal signal leakage. If we specify $a>0.5$ threshold for a true value, the result can be considered to be false.

In Figure $7 \mathrm{~B}, \mathrm{Y} 1$ is preset as 1 , and $\mathrm{X} 1$ is preset as 0 . The circuit functions as $(\mathrm{Y} 2+\mathrm{Y} 3) \cdot \mathrm{X} 2$. For this computing pattern, input combinations of $(1+0) \cdot 1,(0+1) \cdot 1$, and $(1+1) \cdot 1$ return true. The other five combinations of inputs, $(0+0) \cdot 0,(1+$ $0) \cdot 0,(0+1) \cdot 0,(0+0) \cdot 1$, and $(1+1) \cdot 0$, return false. As shown in the figure the reaction rate is the highest for the system with all three true inputs. Here, the reactions are monitored for $12 \mathrm{~h}$. Within this time the fluorescence intensity of the other two true output systems reaches $75 \%$ of that of the highest output, thus representing successful true outputs. The remainder of the operations yield different levels of fluorescence intensities all below 0.3 , thus can be considered to be false outputs.

In Figure $7 \mathrm{C}, \mathrm{Y} 1$ is preset as 0 , and $\mathrm{X} 1$ is preset as 1 . The circuit functions as $\mathrm{Y} 2 \cdot \mathrm{Y} 3+\mathrm{X} 2$. Five combinations of inputs of this circuit return true, and the other three combinations return false. The combinations leading to the true output are $0 \cdot 0+1$, $1 \cdot 1+0,1 \cdot 0+1,0 \cdot 1+1$, and $1 \cdot 1+1$. Among the five true outputs, three reactions are relatively fast. The fastest reactions finish in approximately $4 \mathrm{~h}$, while the two slower reactions reach $70 \%$ intensity (of the fastest) in $12 \mathrm{~h}$. The operations with false outputs all plateaued below 0.3.

The final computing pattern of the circuit is $\mathrm{Y} 2+\mathrm{Y} 3+\mathrm{X} 2$, which can be realized by presetting input $\mathrm{Y} 1$ and $\mathrm{X} 1$ both as 1 (Figure 7D). If any input among $\mathrm{Y} 2, \mathrm{Y} 3$, and $\mathrm{X} 2$ is true, the circuit returns true. Indeed, only $0+0+0$ returns a false output. Five input combinations that have at least one true input from the second generation gate, or both true inputs from the first generation gate, have similar kinetics and produce final fluorescence intensities between 1.0 and 1.1, representing a true output. The fluorescent intensity of the curves corresponding to the other two cases (with a true input from only one of the first majority gates) plateaus at 0.6 in $12 \mathrm{~h}$ with slower kinetics, and also represents a true output.

The 4 plots shown in Figure 7 demonstrate that the signal leakage of each false computation pattern is controlled below $30 \%$. The true outputs all reach intensities higher than $60 \%$. This suggests that the two-generation logic gate cascade is functioning properly. However, some reactions are obviously slower and result in lower intensities than others. Generally, the more true inputs (including the controlled two preset inputs, $\mathrm{Y} 1$ and $\mathrm{X} 1)$ in a system, the faster the reaction is. For example, in Figure $7 \mathrm{~B},(1+1) \cdot 1$ is faster than both $(1+0) \cdot 1$ and $(0+$ $1) \cdot 1$. In addition, if the true output depends on a true intermediate transferred from the first generation $\left(M_{Y}\right)$ to the second generation $\left(M_{X}\right)$, the reaction is slower. The different rates of each computation reaction can be easily explained. The intermediate that is transferred from $M_{Y}$ to $M_{X}$ is within the circular strand of the $M_{Y}$ Calculator. Its exposure induces the strand displacement reaction between the intermediate segment in the middle of the circular strand on $\mathrm{M}_{\mathrm{Y}}$ and the strands bound to the circular $M_{X}$ Calculator to expose the final output. Both circular structures in this step experience a crowded physical environment for the reaction, thus slowing down the strand displacement reaction in the $\mathrm{M}_{\mathrm{X}}$ Calculator.

The main source of leakage of the system is the cross talk between the two generations of outputs. Specifically, the three inputs of $\mathrm{M}_{\mathrm{Y}}$ all contain the entire sequence of the intermediate between $M_{Y}$ and $M_{X}$, except the toehold. A ssDNA domain can displace the identical domain of a dsDNA, although the reaction rate is magnitudes lower than toehold directed strand displacement. $^{9,10}$ The inputs of $\mathrm{M}_{\mathrm{Y}}$ can displace the X3* strand in $M_{X}$. Thus, when there are inputs of both generations present at the same time and the output should be 0 , there is a possibility of leakage. Here we used a higher concentration of the first generation than the second generation so that ratio of the reaction rates of the toehold-directed strand displacement and the undesired nontoehold-directed reaction is higher. In preliminary experiments, the molar ratio of $M_{Y}$ to $M_{X}$ was $1: 1$. The outstanding leakage was approximately $50 \%$. In later experiments and in Figure 7 the molar ratio of $M_{Y}$ to $M_{X}$ is 2:1. Here, the leakage is well-controlled down to below $30 \%$.

Conclusion. We experimentally realized a three-input majority gate based on enzyme free DNA strand displacement reactions. A three-input majority gate is a basic and a versatile logic gate that can be switched between OR and AND gates. The circular structural design presented here provides a new route for designing complex logic gates and may serve as an efficient candidate in designing efficient DNA computing circuits. By combining two three-input majority gates in series, we realized a multifunctional circuit that can be employed in four different forms according to the demand.

Although our design does require a change in the length of strands (which may cause slower reaction kinetics) when scaling up computing circuits, it still provides an alternative strategy for constructing complex circuits. Due to the nature of our majority gate where the inputs and outputs are all ssDNA, it is foreseeable that a circular logic gate can be combined with other existing DNA logic gates ${ }^{13,16}$ for construction of larger circuits for more advance computation.

\section{ASSOCIATED CONTENT}

\section{S Supporting Information}

Detailed descriptions of DNA logic gate design, materials and methods, and additional data analysis. This material is available free of charge via the Internet at http://pubs.acs.org.

\section{AUTHOR INFORMATION}

\section{Corresponding Author}

*E-mail address: yan_liu@asu.edu; weili6@asu.edu.

\section{Notes}

The authors declare no competing financial interest.

\section{ACKNOWLEDGMENTS}

This work was supported by grants from the Army Research Office; Office of Naval Research; National Science Foundation; and Department of Energy to Y.L. Y.L. is also supported by the Technology and Research Initiative Fund from Arizona State University.

\section{REFERENCES}

(1) Liu, X.; Yan, H.; Liu, Y.; Chang, Y. Small 2011, 7, 1673.

(2) Pinheiro, A. V.; Han, D.; Shih, W. M.; Yan, H. Nat. Nanotechnol. 2011, 6, 763

(3) Adleman, L. M. Science 1994, 266, 1021.

(4) Stojanovic, M. N.; Stefanovic, D. Nat. Biotechnol. 2003, 21, 1069.

(5) Elbaz, J.; Lioubashevski, O.; Wang, F.; Remacle, F.; Levine, R. D.; Willner, I. Nat. Nanotechnol. 2010, 5, 417.

(6) Benenson, Y.; Gil, B.; Ben-Dor, U.; Adar, R.; Shapiro, E. Nature 2004, 429, 423.

(7) Yurke, B.; Turberfield, A. J.; Mills, A. P.; Simmel, F. C.; Neumann, J. L. Nature 2000, 406, 605.

(8) Turberfield, A. J.; Mitchell, J. C.; Yurke, B.; Mills, A. P., Jr.; Blakey, M. I.; Simmel, F. C. Phys. Rev. Lett. 2003, 90, 118102.

(9) Yurke, B.; Mills, A., Jr. Genet. Program Evolvable Mach. 2003, 4, 111. 
(10) Zhang, D. Y.; Winfree, E. J. Am. Chem. Soc. 2009, 131, 17303.

(11) Soloveichik, D.; Seelig, G.; Winfree, E. Proc. Natl. Acad. Sci. 2010, 107, 5393.

(12) Phillips, A.; Cardelli, L. J. R. Soc. Interface 2009, 6, S419.

(13) Seelig, G.; Soloveichik, D.; Zhang, D. Y.; Winfree, E. Science 2006, 314, 1585.

(14) Zhang, D. Y.; Turberfield, A. J.; Yurke, B.; Winfree, E. Science 2007, 318, 1121.

(15) Yin, P.; Choi, H. M. T.; Calvert, C. R.; Pierce, N. A. Nature 2008, 451, 318.

(16) Qian, L. L.; Winfree, E. Science 2011, 332, 1196.

(17) Qian, L. L.; Winfree, E.; Bruck, J. Nature 2011, 475, 368.

(18) Imre, A.; Csaba, G.; Ji, L.; Orlov, A.; Bernstein, G. H.; Porod, W. Science 2006, 311, 205. 